\title{
Tracheotomien vor 1800
}

\section{Über 55 erfolgreiche Fälle und ihre Indikationen}

Von Karin Biefel und Wolfgang Pirsig

Bis 1800 galt die Tracheotomie als riskanter, mit einer hohen Mortalität behafteter, mutiger Schritt, der nur im äußersten Notfall bei drohender Erstickungsgefahr durch Verlegung der Luftwege angewendet wurde. Erst im 19.Jahrhundert gelang es, die Tracheotomie zu einem chirurgischen Routineverfahren zu entwickeln. Hierzu trugen vor allem Pierre Fidèle Bretonneau aus Tours (1778-1862) und sein Schüler Armand Trousseau aus Paris (1801-1867) bei, welche den Luftröhrenschnitt fast regelmäßig bei atembedrohender Diphtherie und Croup ausführten $(10,65,66)$.

Seit dem 18. Jahrhundert sind mehrere historische Zusammenstellungen und operationstechnische Anleitungen zur Tracheotomie erschienen (Übersicht bei 6,50). Mit der Entwicklung des Luftröhrenschnittes befaßten sich vor allem Schuchardt (59) und Goodall (26), die aus der Literatur insgesamt über 30 erfolgreiche Tracheotomien bis 1800 gesammelt haben. Eine exakte Zahlenangabe ist nicht möglich, da in der Aufstellung von Schuchardt mehrere, zahlenmäßig nicht benannte Luftröhrenschnitte von Severino (61) enthalten sind. Wir fanden beim Studium der alten Quellen Berichte über 25 weitere lebensrettende Laryngotracheotomien, so daß wir im folgenden von über 55 Operationen berichten können, die speziell unter dem Gesichtspunkt der Indikation diskutiert werden.

Die Bezeichnung «tracheotomia» für die operative Eröffnung der Luftwege in der Halsregion taucht erstmalig 1649 bei Thomas Fienus aus Löwen (1567-1631) in der vierten Ausgabe seines Buches «Libri chirurgici XII» (20) auf. Obwohl sich auch Lorenz Heister aus Helmstedt (1683-1758) in den seinerzeit in Europa sehr bekannten «Institutiones chirurgicae» (29) für diesen Terminus einsetzte, wurde der Begriff erst Anfang des 19. Jahrhunderts durch Bretonneau (11) geläufig. Bis dahin wurde die Operation häufig als Bronchotomie, seltener als Laryngotomie oder Laryngotracheotomie bezeichnet. Der deutsche Ausdruck «Luftröhrenschnitt/-eröffnung» ist schon 1666 bei Johannes Scultetus aus UIm (1595-1645) in der deutschsprachigen Ausgabe «Wund-Artzneyisches Zeug-Haus» (60) zu lesen. In diesem Buch findet sich auch eine Reihe von fünf Holzschnitten (Abb.5), auf denen 
der Vorgang der Tracheotomie bei einem erstickenden Menschen bis zur befreiten Atmung durch die eingelegte Trachealkanüle erstmals wie in einer modernen Operationslehre in einer Bilderfolge wiedergegeben ist.

\section{Älteste Hinweise auf Tracheotomien}

Dokumente über die Tracheotomie finden sich seit etwa 5000 Jahren. Einzelne Autoren $(11,73)$ interpretieren bestimmte Darstellungen auf ägyptischen Tafeln aus Abydos und Saqqara aus der Zeit um 3000 v. Chr. als Abbildungen einer Tracheotomie (Abb.1). Von den drei hier gezeigten Personen wird der rechts sitzenden ein Messer an den Hals gesetzt, das von der mittleren Person geführt wird. Der links stehende Mensch reicht der mittleren Person etwas an. Die mittlere Figur wird als Arzt gedeutet, der eine Tracheotomie durchführt. Daß hier keine Tötungsszene dargestellt wird, läßt sich aus der Anhäufung der vier «Anch»-Zeichen über dem Kopf des «Tracheotomierten» ablesen. Das «Anch»-Zeichen ist die Hieroglyphe für Leben.

Schriftlich erwähnt wird die Tracheotomie erst im ersten vorchristlichen Jahrhundert: Claudius Galenus (130-200 n. Chr.) betrachtet Asclepiades

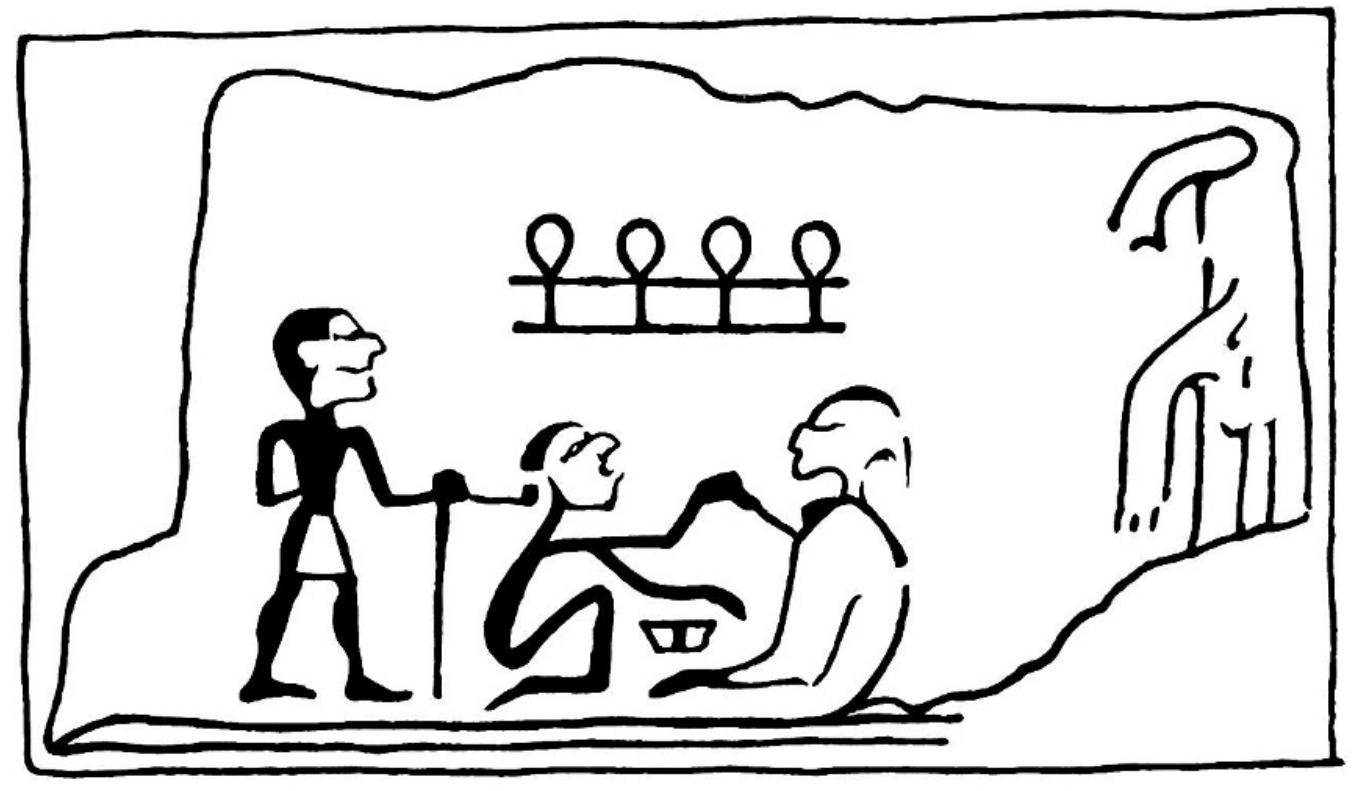

Abb. 1: Ahas Steinplatte aus Abydos (1. Dynastie) mit der diskutierten Darstellung einer Tracheotomie (73) 


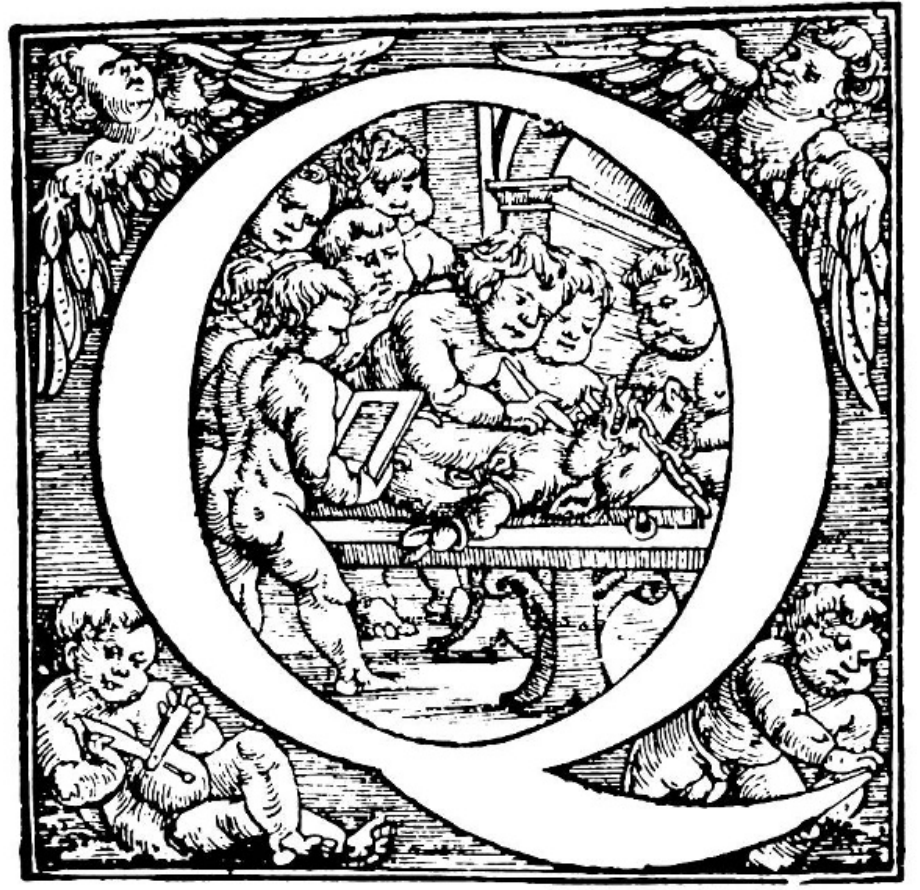

Abb. 2: Tracheotomie bei einem Schwein in Vesals «De humani corporis fabrica» 1555 (69)

von Prusa in Bithynien (124-56 v. Chr.) als den Erfinder der Tracheotomie, um die Cynanche zu behandeln: «Asclepiades ultimum auxilium posuit in iis qui maxime suffocantur laryngem incidere» (23). Aus Galens Schriften geht jedoch nicht hervor, ob Asclepiades die Operation auch selbst ausführte.

Im Talmud, dem jüdischen Sammelwerk aus dem 2. Jahrhundert v. Chr. bis zum 5. Jahrhundert n. Chr., ist die Geschichte von der Beatmung und Rettung eines Mutterschafes mit einem Schilfrohr in der Trachea nach Halsverletzung festgehalten (33).

Eine genaue Beschreibung der Indikation zur Tracheotomie und des Procedere der Operation ist erst von Antyllus, der um die Mitte des 2. Jahrhunderts n. Chr. zu Kaiser Hadrians Zeiten lebte, über Paulus von Aegina (607-690) überliefert. Antyllus gab für die Inzision der Trachea dieselbe Stelle an, die auch heute noch als optimal angesehen wird: zwischen dem dritten und vierten Trachealring (46).

Bis zum Mittelalter liegen von den griechischen, römischen und arabischen Ärzten zwar zahlreiche Diskussionen, jedoch keine Berichte über durchgeführte Tracheotomien vor (59). 
Die erste Abbildung eines Luftröhrenschnittes findet sich 1555 in Vesals «De humani corporis fabrica libri septem» innerhalb der Initiale «Q» bei einem Schwein (Abb.2). In diesem Experiment führte Vesal durch die Halswunde ein Schilfrohr in die Trachea des Tieres ein und erhielt das Schwein am Leben, indem er es über das Rohr atmen ließ (69).

\section{Erste erfolgreiche Tracheotomie: 1546 n. Chr.}

Erst 1546 wurde von Antonio Musa Brasavola aus Ferrara (1490-1554) über einen erfolgreichen Luftröhrenschnitt bei einem Menschen publiziert, der an Angina zu ersticken drohte (9).

Nach Iacobus Hollerius Stempanus (1504-1562) ließ sich ein Anginakranker in größter Atemnot die Trachea ohne Arzt eröffnen und wurde gerettet (32).

Erstmals abgebildet wurde ein Luftröhrenschnitt an einem Menschen von Julius Casserius (1545-1616), Professor der Anatomie in Padua (Abb. 3).

Marco Aurelio Severino aus Tursi (1580-1656) soll nach Kühn (37) und Schuchardt (59) in den großen Diphtherieepidemien, die seit 1616 in Neapel herrschten, mehrfach mit Erfolg tracheotomiert haben.

Nicolas Habicot aus Paris (1550-1624) schilderte 1620 sehr anschaulich vier Fälle lebensrettender Tracheotomien (Abb.4). Zwei waren wegen Schnittverletzungen im Halsbereich und eine wegen Zerschmetterung des Larynx und des oberen Anteiles der Trachea durch einen Büchsenschuß durchgeführt worden. Einem 14jährigen Jungen, der an einem Fremdkörper in der Speiseröhre zu ersticken drohte, rettete Habicot mit der «Bronchotomie» nicht nur das Leben. Er gab dem Jungen auch die neun in ein Leintuch eingewickelten Goldmünzen zurück, die dieser aus Angst vor Dieben zu schlucken versucht hatte. Habicot stieß die Münzen mit einer Sonde aus Blei in den Magen hinab, so daß das Gold nach einigen Tagen auf natürlichem Wege wieder zum Vorschein kam (27).

Nicolaus Fontanus aus Amsterdam berichtete 1641 über eine erfolgreiche Tracheotomie bei Angina (22), Réne Moreau aus Paris (1587-1656) über zwei weitere Fälle (44).

Nach Thomas Bartholin (1616-1680) hat Adrian van den Spieghel aus Brüssel (1578-1625), Professor der Anatomie in Padua, die «Laryngotomie» mit glücklichem Ausgang bei einer Frau aus Padua durchgeführt (2). Wie 


\section{TAB. XXII. DE LARYNGOTOMIA.}

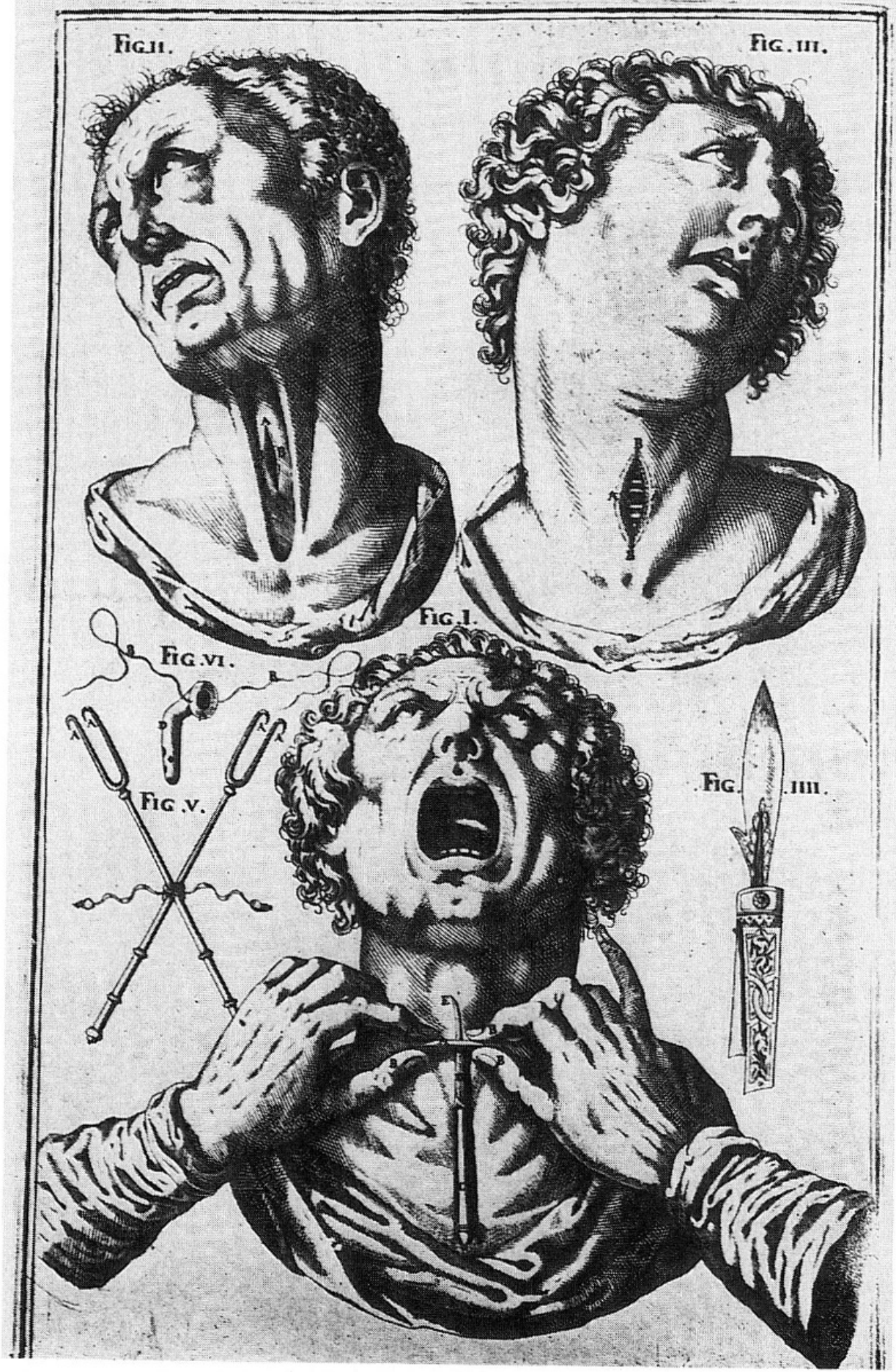

Abb. 3: Darstellung einer Tracheotomie beim Menschen in Casserius «De vocis auditusque...» 1600 (12) 


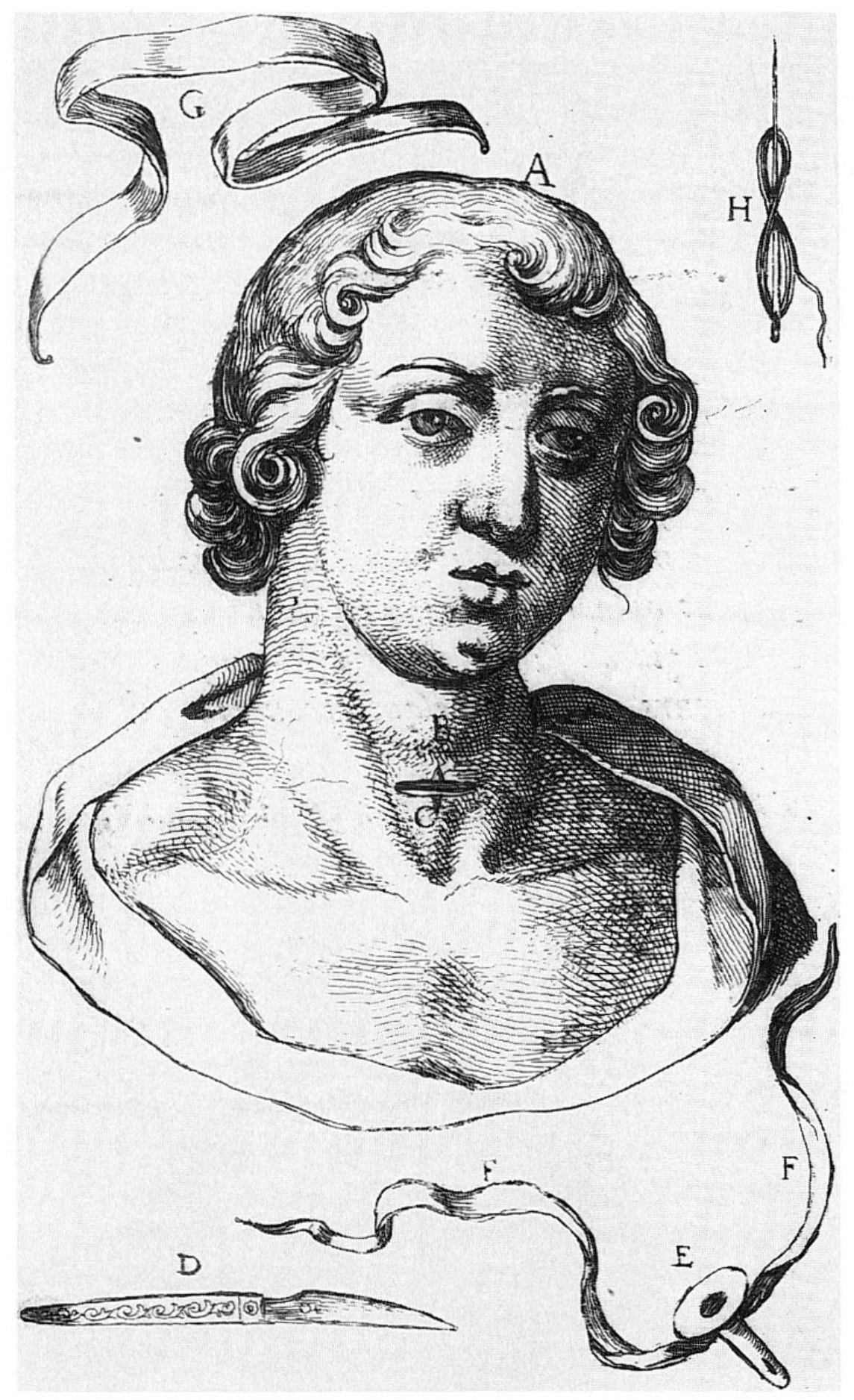

Abb.4: Tracheotomierter Patient bei Habicot, 1620 (27) 

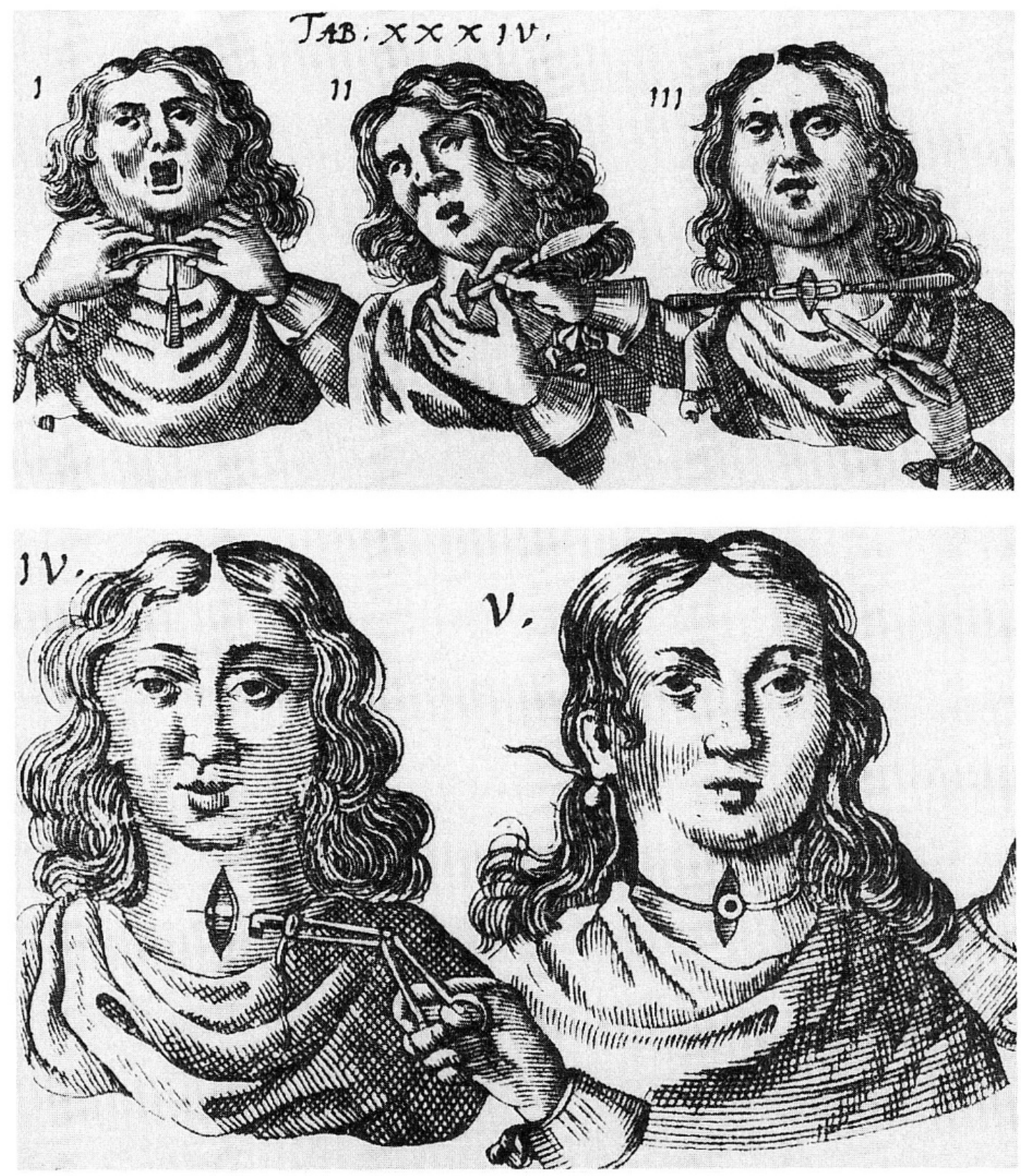

Abb. 5: (A, B): Ablauf einer Tracheotomie bei Scultetus 1666 (60). Als Vorlage zu dieser Tafel XXXIV diente wahrscheinlich die Abbildung von Casserius

bereits Louis (40) und Schuchardt (59) versicherten, hat jedoch der Operateur hiervon in seinen eigenen Schriften nichts festgehalten. 
Lazare Meyssonnier aus Lyon (1602-1672) schrieb 1664, er habe mit der Tracheotomie drei Menschen gerettet, die an «Angina» bzw. «Squinance» zu ersticken drohten (15).

Matthäus Gottfried Purman aus Breslau (1648-1721) nahm 1672 einen heilenden Luftröhrenschnitt «wegen großer Inflammation und Geschwulst des Halses und Schlunds» vor (53).

Smalsius, ein Chirurg aus Leiden, soll nach Louis (40) und Schuchardt (59) einen Mann erfolgreich «bronchotomiert» haben, wie Stephan Blankaart aus Amsterdam (1650-1702) in seinen «Collectanea medico-physica» (7) berichtet. Wir konnten die zitierte Tracheotomie in Blankaarts Werk nicht finden.

1694 benutzte Fulvio Gherli aus Modena nach einer lebensrettenden Tracheotomie bei einer Frau mit «Angina spuria» eine Trachealkanüle aus Blei, die er vier Tage in der Wunde beließ (25).

Jean-Baptiste Verduc aus Paris berichtete in seinen Werken über zwei Luftröhrenschnitte, 1701 bei «Cynanche» und 1712 zur Entfernung eines trachealen Fremdkörpers $(67,68)$.

1720 schrieb René-Jacques Croissant de Garengeot aus Paris (1688-1759) über eine Tracheotomie bei Bräune (24) und bildete die Operationssituation in zwei dekorativen Holzschnitten ab (Abb.6).

\section{Tracheotomie wegen Fremdkörperaspiration}

Im gleichen Jahr entfernte Heister über einen Luftröhrenschnitt einen Fremdkörper aus der Trachea (30). 1739 schilderte er im Anschluß an seinen eigenen Fall eine gelungene Tracheotomie seines Kollegen Johann Jacob Rau aus Leiden (1658-1719), der ebenfalls einen Fremdkörper extrahiert hatte (29).

1730 publizierte George Martin (1702-1743) über vier weitere erfolgreiche Luftröhrenschnitte. Einen dieser Eingriffe hatte er selbst bei einem jungen Mann mit laryngealer Angina durchgeführt. Die Operateure in den übrigen drei Fällen waren Dr. Oliphant in Gask in Perthshire, Baxter, ein Chirurg in Coupar of Fife und ein namentlich nicht bekannter Chirurg gewesen (41).

Abenteuerlich klingt die Geschichte der Tracheotomie, die 1733 in der französischen Zeitschrift «Le Pour et Contre» mitgeteilt wurde (35): Der junge Londoner Chirurg Chovell setzte einen Gedanken des Paduaner 


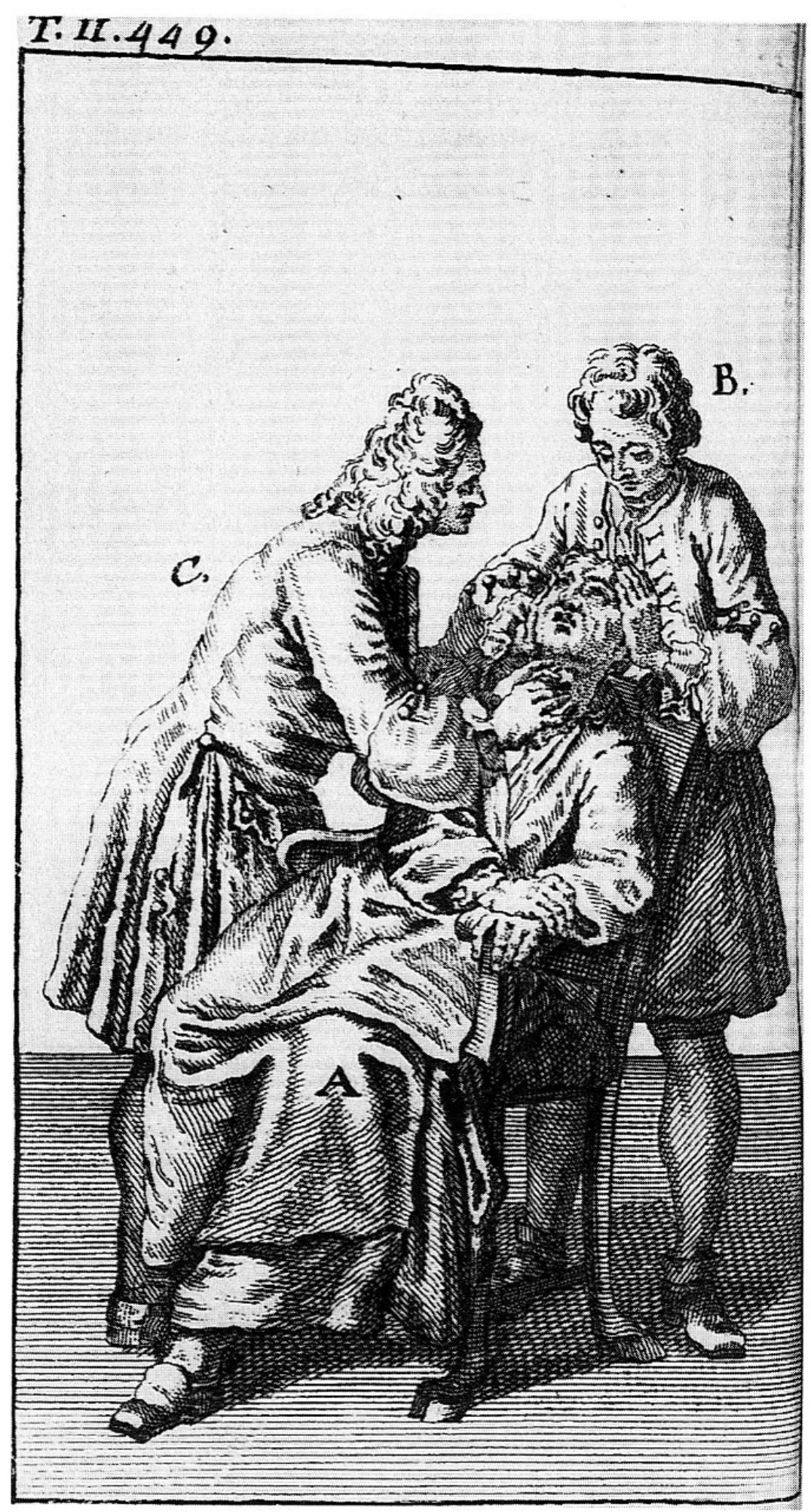

Abb.6: Tracheotomie am sitzenden Patienten bei Garengeot 1731 (24) 
Professors Santorio Santorio (1561-1636) aus dem Jahre 1625 in die Tat um. Santario hatte geschrieben, daß die Tracheotomie auch die zum Erhängen Verurteilten vor dem Ersticken bewahren könnte, wenn es erlaubt wäre, sie anzuwenden: «... imo si illa (= die Tracheotomie) uti liceret in laqueo interrimendis ipsos quoque a suffocatione praeseruaret» (57). Chovell tracheotomierte den zum Tode durch den Strang verurteilten Fleischer namens Gordon vor dessen Hinrichtung heimlich im Gefängnis. Bald nach Vollstreckung des Urteils war der totgeglaubte Mann seinen Angehörigen übergeben worden, die ihn zu Chovell brachten. Gordon war noch am Leben und öffnete nach verschiedenen «Wiederbelebungsmaßnahmen» sogar noch einmal die Augen, ehe er einen tiefen Seufzer ausstieß und verstarb.

Die Tracheotomie, die Pedro Virgili aus Cadiz (1699-1776) wegen einer Entzündung des Larynx und Pharynx (esquinancie) durchführen mußte, verlief aufgrund einer starken Blutung sehr dramatisch, wie aus seiner Publikation von 1743 hervorgeht (74).

1748 propagierte Felix Bauchot, der als Chirurg in Port Louis arbeitete, die Tracheotomie mit einem geraden Bronchotom, d.h. einer kurzen Lanzette, die sich in einer platten silbernen Scheide bewegt. Mit diesem Instrument hat er zwei Patienten vor dem Tod durch «esquinancie» bewahrt $(3,4)$.

Durch eindrucksvolle Holzschnitte (Abb.7) ist der Luftröhrenschnitt belegt, den Johann Michael Engel in Augsburg zur Extraktion eines Fremdkörpers aus der Trachea durchführen mußte, wie er 1750 mitteilte (18).

1765 publizierte Mersenne aus Mansigné in Maine/Frankreich über eine Tracheotomie, die er bei einer Frau im siebten Monat ihrer Schwangerschaft wegen eines «violent mal de gorge» vorgenommen hatte. Anstelle einer Metallkanüle hatte er hierbei eine Gänsefeder benutzt (43).

\section{Tracheotomie zur Reanimation}

Philippe Nicolas Pia (1721-1799) berichtete 1776 sehr spannend über einen interessanten Fall: 1766 war ein 33jähriger Mann wegen Straßenraubes gehängt worden. Als er nach 29 Minuten vom Strang abgeschnitten worden war, nahmen sich Mediziner seiner an. Sie hatten sich eingefunden, um an dem Gehängten verschiedene Wiederbelebungsmaßnahmen zu erproben. Sie begannen damit, den Mann mit Reizmitteln einzureiben und Tabaks- 
klistiere zu setzen. Nach zwei Stunden führte einer der Mediziner namens Glover die Tracheotomie durch und insufflierte durch eine Kanüle. Der Delinquent erholte sich hierauf rasch und konnte bereits am folgenden Tage die Flucht vor der Justiz ergreifen, die ihn wieder zurückholen wollte, wie gerüchteweise verlautete (49).

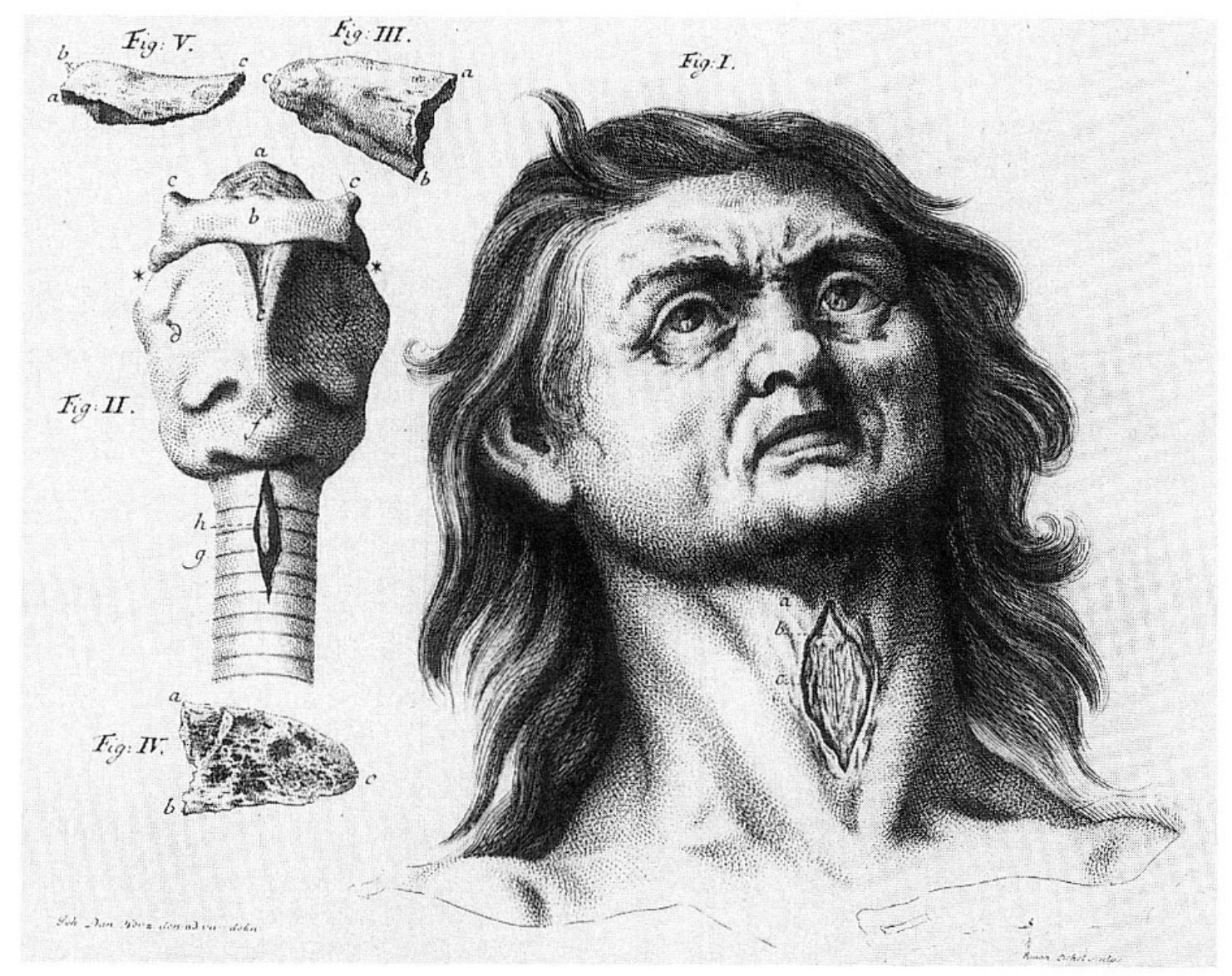

Abb. 7: Tracheotomiedarstellung aus Engels «Sendschreiben...» 1750 (18)

Gerardus Blasius (1626?-1692) berichtete 1666, daß ein gewisser Cl. Patinus in Paris in drei Fällen die erfolgreiche Durchführung einer Tracheotomie sah (70). 
Auch Vidal aus Guerrande verwendete wie Mersenne mit Erfolg einen Federkiel bei der «Bronchotomie», die er 1771 bei einem Mann wegen einer «esquinancie inflammatoire et suffocante» vornahm (72).

1774 publizierten sowohl Germain- Picaut de la Martinière (1696-1783) aus Paris (42) als auch Friedrich Wendt (1738-1818) aus Erlangen (75) über je eine lebensrettende Tracheotomie zur Entfernung eines trachealen Fremdkörpers.

August Gottlieb Richter aus Göttingen (1742-1812) modifizierte das Bronchotom von Bauchot, indem er Trokart und Kanüle länger machte und beiden die Krümmung eines Viertelkreises gab. Wie er 1776 mitteilte, erprobte er dieses Instrument jahrelang mehrfach an Hunden und an Toten, ehe er es «sicher und leicht» an einem lebenden Menschen anwenden konnte (55). Die gekrümmte Kanülenform von Richter wurde das Muster aller modernen Kanülenformen, nachdem 1730 Martin (41) schon das Prinzip der Doppelkanüle beschrieben hatte.

Ein Federkiel diente Ferriere aus Mouy in Beauvoisis wiederum als Kanüle, als er 1779 erfolgreich wegen einer «angine oedémateuse» tracheotomierte (19).

1782 rettete John Andrew aus London mit einem Luftröhrenschnitt das Leben eines fünfjährigen Knaben, der an «cynanche trachealis» litt (1).

Nachdem Georg Detharding aus Rostock (1671-1747) bereits 1714 den Vorschlag gemacht hatte, Ertrunkenen durch die Tracheotomie das Leben wiederzugeben (14), erschien 1783 erstmals eine Veröffentlichung über einen gelungenen Luftröhrenschnitt bei dieser Indikation von Claude Pouteau aus Lyon (1725-1775) (51). Benjamin Bell (1749-1806) schrieb ein Jahr später über zwei weitere Fälle (5). Darüber hinaus berichtete er über eine geglückte Tracheotomie bei starker Pharynxschwellung, die durch hochdosierte Quecksilbergabe verursacht war.

Pouteau gab mit seiner Empfehlung, die Tracheotomie bei Ertrunkenen vorzunehmen, um das in der Luftröhre befindliche Wasser durch das Trachealröhrchen abzusaugen und anschließend warme Luft in die Lungen einzublasen, erstmals eine Anleitung zur Bronchialtoilette. In o.g. Fall war zwar die künstliche Beatmung durchgeführt worden, die Bronchialtoilette allerdings noch nicht (51).

Petit entfernte 1786 über einen Luftröhrenschnitt einen trachealen Fremdkörper (48).

Philippe-Jean-Josephe Pelletan aus Paris (1747-1829) publizierte 1810 über sechs erfolgreiche Tracheotomien bei Fremdkörpern in der Luftröhre, 
von denen mindestens zwei aus der Zeit vor 1800 stammen. Eine dritte aus dem Jahre 1788 gilt als die erste Laryngofissur in der Medizingeschichte. Sie war zur Extraktion eines im Kehlkopf eingeklemmten Sehnenstückes vom Kalb vorgenommen worden (47).

John Hunter aus London (1728-1793) führte 1792 eine lebensrettende Koniotomie bei «Bräune» aus (34). Er wendete damit ein Operationsverfahren an, das schon 1776 von Félix Vicq d'Azyr (1748-1794) entdeckt und an Hunden entwickelt worden war (71). 1793 berichtete Lerz über eine Tracheotomie, die er zur Entfernung einer Bohne aus den Luftwegen eines fünfjährigen Mädchens vorgenommen hatte (38).

Guiseppe Flajani aus Rom (1741-1808) beschrieb im ersten Band seiner «Collezione d'osservationi e riflessioni de chirurgia» (1797) sehr anschaulich eine erfolgreiche Tracheotomie bei Angina (21).

Christian Karl Klein aus Stuttgart (1772-1825) führte die Operation 1799 (36) ebenso wie Rammelsberg in Blankenburg am Harz im Jahre 1800 (54) an einem Kind zur Extraktion eines trachealen Fremdkörpers aus. In beiden Fällen konnten die kleinen Patienten durch den Eingriff geheilt werden.

\section{Übersicht der Indikation zur Tracheotomie}

Fassen wir die Indikationen zu den über 55 erfolgreichen Tracheotomien bis 1800 zusammen, so ergeben sich folgende Zahlen: über 23mal Entzündungen im Pharynx- und Larynxbereich, 14mal Fremdkörper, dreimal Ertrinken, dreimal Halsverletzungen, zweimal Ersticken durch Erhängen und einmal eine toxische Schwellung im Halsbereich. In neun Fällen sind die Indikationen nicht erwähnt.

Entzündungen im Pharynx- und Larynxbereich, geläufig unter dem Terminus «cynanche» bzw. «angina», verdienen nicht nur als häufigste Indikation zu lebensrettenden Luftröhrenschnitten bis 1800 Beachtung. Gleichzeitig sind sie auch die Affektionen, bei denen die Tracheotomie im ersten vorchristlichen Jahrhundert vorgeschlagen (23) und dann mehr als eineinhalb Jahrtausende später auch erstmalig mit Erfolg durchgeführt wurde (9).

Diese Rachen- und Kehlkopfentzündungen bilden somit den Grundstein des Indikationskataloges der Tracheotomie.

1620 erweiterte sich dieser Katalog um Schnitt- und Schußwunden des 
Halses sowie um Fremdkörper in Luft- und Speiseröhre (27). 1625 (57) bzw. 1733 (35) kommen die prophylaktischen Tracheotomien zur Verhinderung des Todes durch Erhängen dazu. Zur Rettung Ertrunkener wurde die Tracheotomie erstmals 1714 vorgeschlagen (14) und 1783 erfolgreich in die Tat umgesetzt (51). 1740 stellte Samuel Sharp (1700-1778) die Verletzung der Atemwege durch eine Struma, die man damals «Bronchocele» nannte, klar als Indikation zur Tracheotomie heraus (62). Bis zum Ende des 18. Jahrhunderts waren die heute sogenannten «klassischen» Indikationen zur Tracheotomie im wesentlichen auch schriftlich fixiert. Dies belegen die Indikationskataloge jener Zeit $(5,13,17,56)$, deren ausführlichster von Richter aus dem Jahre 1798 stammt. Im Originaltext über mehrere Seiten kommentiert (von uns in Tabelle 1 zusammengefaßt) und listet er die klassischen Indikationen zum Luftröhrenschnitt nahezu wie heute auf. Zwischen Indikationsstellung und Ausführung der Tracheotomie, ersichtlich aus Richters Indikationsliste und unserem Überblick, scheint bis 1800 eine erhebliche Diskrepanz zu bestehen. Allerdings ist hierbei zu bedenken, daß über erfolglose Tracheotomien im Schrifttum aus verständlichen Gründen nur sehr selten berichtet wurde $(13,47,59,62)$. Die genauen Zahlen aller Tracheotomien bis 1800 werden wir wohl nie mehr eruieren können. In seiner Sammelstatistik bis 1860 registrierte Kühn (37) 708 Tracheotomien mit günstigem oder tödlichem Ausgang. 1891 hatten Prescott und Goldthwait (52) schon 23941 Luftröhrenschnitte allein wegen obstruierender Diphtherie mit einer Heilungsquote von $28,67 \%$ aus dem Schrifttum der Welt zusammengetragen. Heute dürften nur aus dem letzten Jahrzehnt weltweit sicher einige hunderttausend Tracheotomien zusammenkommen.

Wie groß die Furcht der Ärzte vor der Tracheotomie noch vor zwei Jahrhunderten war, verdeutlicht eindrucksvoll der tragische Tod von George Washington 1799. An der Schwelle zu einer neuen Ära in der Geschichte der Tracheotomie starb der erste Präsident der Vereinigten Staaten von Amerika im Alter von $67 \mathrm{Jahren}$ an einer entzündlichen Kehlkopfkrankheit, nach neueren Erkenntnissen wahrscheinlich an einer Epiglottitis acutissima. Von einem der drei behandelnden Ärzte war zwar bei der Diskussion um die Therapie des Kranken der Luftröhrenschnitt vorgeschlagen worden, man hat sich schließlich statt dessen für einen weiteren, vierten Aderla $\beta$ entschieden und dem Patienten damit insgesamt $2000 \mathrm{ml}$ Blut entzogen, ehe dieser nach nur 21 Stunden Krankheitsdauer starb. Niemand hatte in den Vereinigten Staaten von Nordamerika bis dahin gewagt, eine Tracheotomie durchzuführen (58). 
1. Fremdkörper

1.1 Von außen eingetreten in Mund, Rachen, Luftröhrenkopf, Luftund Speiseröhre

1.2 Über die Lungen in die Trachea gelangt

2. Entzündliche Bräune

2.1 Angina inflammatoria laryngea

2.2 Angina tonsillaria

3. Wunden des Luftröhrenkopfes

4. Präoperativ bei Exstirpation «chronischer Geschwülste» im Mund und Rachen, der Fleisch- und Balggeschwülste, der scirrhosen Mandeln und des Rachenpolypen, sowie bei Unterbindung des Polypen der Speiseröhre

5. Heftige Zungenentzündungen

6. Kropfgeschwulst

\section{Literatur}

1 Andrew, J.: zitiert bei (10)

2 Bartholin, T.: De anginia puerorum Campaniae Siciliaeque epidemica Exercitationes; accedit de Laryngotomia Cl.-V.-Renati Moreau, Paris. Lutetiae Parisiorum, Olivarius de Varennes (1646)

3 Bauchot, F.: in: Mém. Acad. roy. Chir. 3 (1757) 12

4 Bauchot, F.: in (40)

5 Bell, B.: A system of surgery. Vol.2, Chap.23: Of Bronchotomy, pp.403-421, London, Edinburgh (1784)

6 Biefel, K.: Die Indikationen zur Tracheotomie: Eine medizingeschichtliche Studie. Dissertation, Ulm (1983)

7 Blankaart, St.: Collectanea medico-physica. Amstelodanus (1680)

8 Boyer, P.: Traité des maladies chirurgicales et des opérations, qui leur conviennent. Ed.5, Tome 5, p.498, Labé, Paris (1846)

9 Brasavola, A.M.: Libros de ratione victus in morbis acutis, Hippocratis et Galeni commentaria et annotationes. Buch IV, Kap. 35, pp.114-115, Scotus, Venetys (1546)

10 Bretonneau, P.F.: Des inflammations spéciales du tissu muqueux, et en particulier de la diphthérite, ou inflammation pelliculaire, connue sous le nom de croup, d'angine maligne, d'angine gangréneuse, etc. Crevot, Paris (1826)

11 Brewer, L. A.: in: Dugan, D. J., P.C. Samson: Tracheotomy: Present day indications and technics. Amer. J.Surg. 106 (1963), pp. 290-306

12 Casserius, J.: De vocis auditusque organis historia anatomica. Lib. I, Cap.20, Tafel 22, Baldinus, Ferrariae (1600) 
13 Desault, P.J.: Euvres chirurgicales publié par Xav. Bichat. Seconde Partie, pp. 213-247, Paris (1798)

14 Detharding, G.: in Steinbach, C.E.: Disputatio medica inauguralis de cynanche, quam praeside rectore magnifico Dn. Georgio Dethardingio etc., pp.15-16, Adler, Rostochii (1723)

15 Dolois, L.G.; L. Meyssonnier: Le Cours de medecine en françois, contenant le miroir de beauté et santé corporelle. Ed. 7, Livre II, Chap.III: De l'Angine ou Squinance, p. 166, Barbier, Lyon (1678)

16 Dumesnil, R.; H.Schadewaldt: Die berühmten Ärzte. Aulis Verlag, Köln (1974)

17 Ehrlich, J.A.: Chirurgische, auf Reisen und vorzüglich in den Hospitälern zu London gemachte Beobachtungen. Bd.1, pp. 77-85, Barth, Leipzig (1795)

18 Engel, J. M.: Sendschreiben an Titulo Pleno Herrn D. Georg Ulrich Schmid, ... Augsburg (1750)

19 Ferriere: Observations sur l'angine céémateuse. J. Méd. Chir. Pharm. (Paris) 62 (1784) 248-253

20 Fienus, T.: Libri chirurgici XII. Tractatus IV: «De Tracheotomia». 4. Auflage, pp.35-38, Goezius, Francofurti (1649)

21 Flajani, G.: Collezione d'osservationi e riflessioni de chirurgia. Bd.1, Osservatione 54, pp. 200-201, Salomoni, Roma (1797)

22 Fontanus, N.: Observationum rariorvm analecta. Cap. III: Vtrum Laryngotomia, in Angina, sit celebranda? Exemplum III, pp.11-15, sumptibus Henrici Laurentii Bibliopolae, Amstelodami (1641)

23 Galenus, C.: Opera omnia. Ed. C.G. Kühn, T.14, pp. 733-734 (Repr. Nachdruck der Ausg. Leipzig 1827)

24 Garengeot, R.-J.C.d.: Traité des operations de chirurgie. Ed.2, Tome 2, p.444, Observation XI, u. p.449, Cavelier, Paris (1731)

25 Gherli, F.: Centuria d'osservazioni rare di medicina, e cirugia. Osservazione vigesima settima. Angina Spuria, pp.96-99, Lovisa, Venezia (1719)

26 Goodall, E. W.: The story of tracheotomy. Brit. J.Child. Dis. 31 (1934) 167-176; 253-272

27 Habicot, N.: Qvestion chirvrgicale, par laqvelle il est demonstré que le Chirurgien doit assurément practiquer l'operation de la Bronchotomie, vulgairement dicte laryngotomie, ou perforation de la fluste ou tuyau du polmon. Iean Corrozet, Paris (1620)

28 Haller, A.v.: Bibliotheca chirurgica. p.498, Olms, Hildesheim - New York (1971)

29 Heister, L.: Institutiones chirurgicae. Vol.2, Pars II, Caput CII. De bronchotomia, laryngotomia seu tracheotomia. Janssonio-Waesbergios, Amstelaedami (1739)

30 Heister, L.: Medicinische, chirurgische und anatomische Wahrnehmungen. pp.10261028, Koppe, Rostock (1753)

31 Hirsch, A.: Biographisches Lexikon der hervorragenden Ärzte aller Zeiten und Völker. 3. Auflage, Urban u. Schwarzenberg, München - Berlin (1962)

32 Hollerius, I.S.: De morborum internorum curatione. Liber I, p. 55, I. Maceus, Parisiis (1567)

33 In: Houlin $57 \mathrm{~b}$ : zit. bei: Simon, I.: L'otorhinolaryngologie hebraique antique et médiévale. Acta Oto-Rhino-Laryngologica Belgica 35, Suppl. IV (1981) 1562-1582

34 Hunter, J.: in (17)

35 Invention nouvelle de l'Art. Le Pour et Contre (Paris) 1 (1733) 154-158 
36 Klein, Ch. K.: Chirurgische Bemerkungen. I. Geschichte eines sehr schweren Luftroehrenschnittes, pp. 168-190, Loefsund, Stuttgart (1801)

37 Kühn, J.E.: Die künstliche Eröffnung der obersten Luftwege. Winter, Leipzig u. Heidelberg (1864)

38 Lerz: Glückliche Operation bei einer in die Luftröhre geschlungenen Bohne. Starks Archiv für Geburtshuelfe, Frauenzimmer- und Neugebohrner Kinder-Krankheiten und Pharmakologie (Jena UCMP), Bd.4, Art. 21, p. 762 (1793)

39 Lichtenthaeler, Ch.: Geschichte der Medizin. 2 Bände, Deutscher Ärzte Verlag, Köln (1974)

40 Louis, A.: Mémoire sur la Bronchotomie. Mém. Acad. roy. Chir. 12 (1768) 201-290

Second Mémoire sur la Bronchotomie, où l'on traite des corps étrangers de la trachée artere. Mém. Acad. roy. Chir. 12 (1768) 292-341

4.1 Martin, G.: The Postscript of a Letter from George Martin, M.D. to William Graeme, M.D.F.R.S. giving an Account of the Operation of Bronchotomy, as it was performed at St. Andrews. Philosophical Transactions Royal Society London, 36 (1730) 448-455

42 Martinière, G.-P.: Observation sur un corps étranger qui perçoit la trachée artère. Mém. Acad. roy. Chir. 14 (1774) 421-426

43 Mersenne: Observation sur la Bronchotomie. J. Méd. Chir. Pharm. 23 (1765) 559-560

44. Moreau, R.: in (2)

45 Morton, L.T.: A Medical Bibliography (Garrison and Morton). Ed.3, Deutsch, London (1970)

46 Paulus von Aegina: Pauli aeginetae medici optimi, Libri septem. Übersetzt von I. Berendes, pp. 495-496, Brill, Leiden (1914)

47 Pelletan, P.J.J.: Clinique chirurgicale. Tome 1, Kap.: Mémoire sur la broncotomie, pp. 1-53, Dentu, Paris (1810)

48 Petit: J. Méd. Chir. Pharm. (Paris) 75 (1788) 376-377

49 Pia, P. N.: Détail des succès de l'établissement que la ville de Paris a fait en faveur des personnes noyées, qui a été adopté dans diverses Provinces de France. Partie 4, pp.177-181, Lottin l'aîné, Paris (1776)

50 Pirsig, W.; H. Rodegra: Chirurgie du larynx et de la trachée. Acta Oto-Rhino-Laryngologica Belgica 35, Suppl. IV (1981) 1427-1504

51 Pouteau, C.: Euvres Posthumes. Tome 2, pp. 189-207, Pierres, Paris (1783)

52 Prescott, W.H.; J.E. Goldthwait: A report of 392 cases of intubation and 139 cases of tracheotomy done at the Boston City Hospital. Boston med. surg. J. 125 (1891) 694-697

53 Purmann, M.G.: Chirurgia Curiosa. Cap. XV: Lufftröhren Oeffnung in der Breune und andern besorglichen Zuständen da der Patient ersticken wil. p.98, Rohrlach, Franckfurt u. Leipzig (1699)

54 Rammelsberg: in: Uhde, C. W. F.: Zusammenstellung der im Herzogthum Braunschweig vom 23. November 1720 bis zum 8. April 1869 ausgeführten Luftröhrenschnitte. Langenbecks Arch. klin. Chir. 11 (1869) 743-761

55 Richter, A.G.: Observationum Chirurgicarum Fasciculus Secundus. Caput III.: De Bronchotomia, pp. 32-43, Dieterich, Gottingae (1776)

56 Richter, A.G.: Anfangsgründe der Wundarzneykunst. Bd.4, pp.224-230, Frankenthal (1798)

57 Santorio, S.: Commentaria in primam fen primi libri canonis Avicennae. pp.362-363, Brogiollus, Venetiis: Sarcinam (1625) 
58 Scheidemandel, H.H.E.: Did George Washington die of Quinsy? Arch. Otolaryngol. 102 (1976) 519-521

59 Schuchardt, B.: Zur Geschichte der Tracheotomie bei Croup und Diphtherie, besonders in Deutschland. Langenbecks Arch. klin. Chir. 36 (1887) 527-604.

60 Scultetus, J.: Wund-Artzneyisches Zeug-Haus. Faksimile-Druck der Scultetus-Ausgabe von 1666, pp. 109-111, Kohlhammer, Stuttgart (1974)

61 Severino, M. A.: zit. bei $(37,59)$

62 Sharp, S.: A Treatise on the operations of surgery. Ed.3, Chap.31: Of Bronchotomy, pp. 185-188, London (1740)

63 Smalsius: zit. bei $(40,59)$

64 Sprengel, K.: Bronchotomie. In: Geschichte der Chirurgie. Teil 1, pp. 177-192, Kümmel, Halle (1805)

65 Trousseau, A.: Nouvelles recherches sur la trachéotomie pratiquée dans la période extrême du croup. Halteste, Paris (1851)

66 Trousseau, A.: Trachéotomie. In: Clinique Médicale de l'Hôtel-Dieu de Paris. Ed.2, Tome 1, pp. 437-453, Baillière, Paris (1865)

67 Verduc, J.-B.: zit. bei $(28,40)$

68 Verduc, J.-B.: Pathologie de la chirurgie dans laquelle on explique toutes les maladies externes du corps humain. Deutsche Übersetzung, Teil 1, p.154, Gleditsch u. Sohn, Leipzig (1712)

69 Vesalius, A.: De humani corporis fabrica libri septem. p. 708; 822-824, Liber VII, Oporinus, Basel (1555)

70 Veslingius, I.: Syntagma anatomicum cum commentariis. Exhibente. Gerardo Blasio. Caput XI, p. 165, Jansonium et Elizoeum, Amstelodami (1666)

71 Vicq d'Azyr, F.: zit. bei $(8,64)$

72 Vidal: Succès de la bronchotomie dans l'esquinancie inflammatoire et suffocante. J. Méd. Chir. Pharm. (Paris) 38 (1772) 358-365

73 Vikentieff, W. pp.90-92 in: «The House of Life», P.Ghalioungui, B. M. Israel, Amsterdam 1973

74. Virgili, P.: Sur une Bronchotomie faite avec succès. Mém. Acad. roy. Chir. 1 (1743) 141-145

75 Wendt, F.: Historia Tracheotomiae nvperrime administratae. Meyer, Vratislavia (1774) 


\section{Zusammenfassung}

Anhand der Originalquellen wird ein kurzer Abriß von über 55 lebensrettenden Luftröhrenschnitten von 1546 bis 1800 gegeben. Die Operation wurde am häufigsten bei Entzündungen im Pharynx- und Larynxbereich und bei Fremdkörpern in Speiseröhre und Trachea angewandt, selten bei Schnitt- und Schußwunden des Halses, bei Ertrunkenen, bei toxischer Schwellung im Halsbereich und zur Verhinderung des Todes durch Erhängen. Bis zum Ende des 18. Jahrhunderts entwickelte sich ein Indikationskatalog für die Tracheotomie, der im wesentlichen schon alle klassischen Indikationen zum Luftröhrenschnitt von heute umfaßte. Seinen Ausgang nahm er bei der Forderung des Asclepiades im ersten vorchristlichen Jahrhundert, die Operation bei Erstickungsgefahr durch Entzündungen im Pharynx- und Larynxbereich auszuführen.

\section{Summary}

Tracheotomies before 1800: More than 55 Successful Cases and their Indications

A short survey is made of more than 55 documented life-saving tracheotomies from 1546 until 1800 by evaluating the original literature. The operation was performed most often because of inflammatory lesions of the pharynx and larynx and to extract foreign bodies from the oesophagus and trachea, rarely for cuts and gunshot wounds of the neck, for the resuscitation of drowned persons, in a case of toxic swelling of the throat and to prevent death by hanging. Until the end of the 18th century a catalogue of indications for tracheotomy had been developed which already included all the classical indications for tracheotomy as nowadays. This catalogue started from the demand of Asclepiades to carry out the operation in cases of impending suffocation by cynanche, the term for any kind of pharyngeal and laryngeal inflammation in the first century B.C.

Dr. med. Karin Biefel

Kreiskrankenhaus

D-7920 Heidenheim

Prof. Dr. med. W. Pirsig

Univ. HNO Klinik

D-7900 Ulm 